\title{
Caractéristiques épidémiocliniques et étiologiques des myélopathies dans le service de neurologie du CHU de Cocody d'Abidjan (Côte-d'Ivoire)
}

\author{
Epidemiological and Etiological Characteristics of Myelopathies in the Neurology Department \\ of the University Hospital Center of Cocody, Abidjan (Ivory Coast)
}

\author{
Z. Mamadou $\cdot$ C. Tanoh $\cdot$ M. Doumbia $\cdot$ M.D. Toudou $\cdot$ M. Amon-Tanoh $\cdot$ C. Yapo-Ehounoud \\ E. Aka-Anghui Diarra
}

Reçu le 16 avril 2018; accepté le 17 septembre 2018

(C) Société de pathologie exotique et Lavoisier SAS 2018

Résumé Le terme de myélopathie définit une souffrance de la moelle épinière quelles que soient les étiologies. Les myélopathies réalisent souvent de véritables urgences thérapeutiques et sont grevées de lourdes séquelles fonctionnelles. L'objectif de ce travail était de décrire les aspects épidémiologiques, cliniques et étiologiques de toutes les myélopathies identifiées lors d'une étude prospective et descriptive qui s'est déroulée du $1^{\mathrm{er}}$ janvier 2015 au 31 décembre 2016 dans le service de neurologie du CHU de Cocody d'Abidjan. Soixante et onze patients sur 1006 ont été inclus, soit une prévalence de $7,06 \%$. Le sex-ratio $(\mathrm{H} / \mathrm{F})$ était égal à 1,7 . L'âge moyen était de 49 ans. Le VIH était associé à la myélopathie dans $24,7 \%$ (17 patients) des cas. Le syndrome de compression médullaire était majoritaire. Le mal de Pott était l'étiologie la plus fréquante des myélopathies compressives (43,9\%), suivi des métastases osseuses (26,8\%). La myélite tuberculeuse était l'étiologie la plus fréquente des myélopathies non compressives (30\%). Les myélites de cause indéterminée représentaient $50 \%$ des cas des myélopathies non compressives.

Mots clés Myélopathie · VIH · Tuberculose · Métastases · Carence en vitamine B12 · Épidémiologie · Étiologies ·

Hôpital $\cdot$ Abidjan · Côte-d'Ivoire · Afrique intertropicale

\section{Z. Mamadou $(\bowtie) \cdot$ C. Tanoh · M. Amon-Tanoh}

C. Yapo-Ehounoud · E. Aka-Anghui Diarra

Service de neurologie, CHU de Cocody,

BP V 13 Abidjan, Côte-d'Ivoire

e-mail : mamadouzakaria1@hotmail.fr

\section{Doumbia}

Service de Neurologie, CHU de Yopougon, Côte d'Ivoire

\section{M.D. Toudou}

Service de médecine interne et spécialités médicales,

Hôpital national de Niamey, Niger

\begin{abstract}
The term myelopathy defines a suffering of the spinal cord whatever the etiologies. They often represent real therapeutic emergencies and are burdened by serious functional sequelae. The aim of this work was to describe the epidemiological, clinical, and etiological aspects of all myelopathies. We have conducted a prospective and descriptive study from January 1, 2015 to December 31, 2016 at the Neurology department of the Cocody University Hospital in Abidjan. Seventy-one patients out of 1,006 were included, that is, a prevalence of $7.06 \%$. The sex ratio was 1.7 . The average age was 49 years old. HIV was associated with myelopathy in $24.7 \%$ of cases. Medullary compression syndrome was predominant. Pott's disease was the etiology of compressive myelopathies (43.9\%) followed by bone metastases (26.8\%). Tuberculous myelitis was the most common etiology of non-compressive myelopathies (30\%). Myelites of undetermined causes account for $50 \%$ of non-compressive myelopathies.
\end{abstract}

Keywords Myelopathy · HIV · Tuberculosis · Metastasis · Lack of vitamin B12 - Epidemiology · Etiologies · Hospital · Abidjan · Ivory Coast $\cdot$ Sub-Saharan Africa

\section{Introduction}

Le terme de myélopathie définit une souffrance de la moelle épinière quelle que soit l'étiologie [6]. Les myélopathies peuvent être classées en myélopathies compressives et en myélopathies non compressives. Elles représentent une urgence diagnostique et thérapeutique, et peuvent donner parfois des séquelles motrices sévères associées à des troubles vésicosphinctériens. Les étiologies sont variables et peuvent être infectieuses, inflammatoires, auto-immunes, 
tumorales, carentielles, toxiques ou vasculaires. L'objectif de ce travail était de décrire les aspects épidémiologiques, cliniques et étiologiques de toutes les myélopathies observées dans le service de neurologie du CHU de Cocody d'Abidjan.

\section{Méthodes}

Nous avons mené une étude prospective et descriptive, réalisée entre le $1^{\mathrm{er}}$ janvier 2015 et le 31 décembre 2016 dans le service de neurologie du CHU de Cocody d'Abidjan. Tous les patients présentant un syndrome médullaire clinique, quels que soient l'âge et le mode d'installation, et ayant bénéficié d'une imagerie médullaire (IRM ou TDM) ont été inclus. Nous avons exclu 20 patients ayant des dossiers incomplets. Le consentement éclairé écrit des patients a été obtenu.

\section{Résultats}

Nous avons inclus 71 patients sur 1006 hospitalisés pendant notre période d'étude ; soit une prévalence hospitalière de $7,06 \%$. Les myélopathies compressives et non compressives représentaient respectivement 57,7 (41 patients) et $42,3 \%$ (30 patients). Le sexe masculin était majoritaire dans $63 \%$ des cas, avec un sex-ratio $(\mathrm{H} / \mathrm{F})$ égal à 1,7. L'âge moyen était de 49 ans, avec des extrêmes allant de 16 à 85 ans. La tranche d'âge $36-45$ ans était majoritaire dans près de $24 \%$ des cas (soit 17 patients).

Le motif de consultation était la faiblesse des membres inférieurs (48 cas), la faiblesse des quatre membres (17 cas) et les troubles de la marche ( 6 cas). La durée moyenne des signes précédant la consultation était de quatre mois, avec des extrêmes allant de 7 jours à 18 mois. Le mode d'installation était essentiellement progressif dans $71,8 \%$ des cas.

La présentation clinique était caractérisée par un syndrome de compression médullaire (56,3\%), un syndrome de section transverse de la moelle $(35,2 \%)$ et une sclérose combinée de la moelle $(5,7 \%)$. Le scanner rachidien a été réalisé dans $53,2 \%$ des cas et l'IRM médullaire dans $64,8 \%$ des cas.

Le VIH était associé à la myélopathie chez 17 (24,7\%) patients : elle était révélatrice chez neuf patients $(9 / 17=52,9 \%)$.

La spondylodiscite à bacille de Koch (BK) et les métastases vertébrales étaient les causes les plus fréquentes des myélopathies compressives dans respectivement 43,9 $(18 / 41)$ et $26,8 \%(11 / 41)$ des cas.

L'adénocarcinome de la prostate était le foyer primitif des métastases chez six patients.

Le mal de Pott siégeait essentiellement au niveau dorsolombaire chez 13 patients (72,2 \%).
La myélite tuberculeuse était la cause la plus fréquente des myélopathies non compressives.

Nous avons retrouvé trois cas de myélopathie par carence en vitamine B12, un cas de neuromyélite optique (NMO) avec des anticorps anti-NMO positifs et un cas d'encéphalomyélite aiguë disséminée (EMAD).

Le tableau 1 décrit les caractéristiques épidémiocliniques et étiologiques de nos patients.

\begin{tabular}{|c|c|c|}
\hline Variables & & Valeurs, n (\%) \\
\hline Sexe & & \\
\hline Hommes & & $45(63,4)$ \\
\hline Femmes & & $26(36,6)$ \\
\hline Sex-ratic & & 1,7 \\
\hline Âge (ans) & & \\
\hline Moyenn & & 49 \\
\hline Extrême & & 16 et 85 \\
\hline $16-25$ & $\begin{array}{l}H=9 \\
F=2\end{array}$ & $11(15,5)$ \\
\hline $26-35$ & $\begin{array}{l}\mathrm{H}=8 \\
\mathrm{~F}=4\end{array}$ & $12(16,9)$ \\
\hline $36-45$ & $\begin{array}{l}H=9 \\
F=8\end{array}$ & $17(23,9)$ \\
\hline $46-55$ & $\begin{array}{l}\mathrm{H}=4 \\
\mathrm{~F}=7\end{array}$ & $11(15,5)$ \\
\hline $56-65$ & $\begin{array}{l}\mathrm{H}=3 \\
\mathrm{~F}=3\end{array}$ & $6(8,5)$ \\
\hline $66-75$ & $\begin{array}{l}H=7 \\
F=2\end{array}$ & $9(12,7)$ \\
\hline $76-85$ & $\begin{array}{l}H=5 \\
F=0\end{array}$ & $5(7,0)$ \\
\hline Étiologies & & \\
\hline Myélopa & compressives & $41(57,7)$ \\
\hline Mal d & & $18 / 41(43,9)$ \\
\hline Métas & & $11 / 41(26,8)$ \\
\hline Tumeu & nidiennes non déterminées & $2 / 41(4,9)$ \\
\hline Myélo & s cervicarthrosiques & $6 / 41(14,6)$ \\
\hline Étiolo & déterminée & $2 / 41(4,9)$ \\
\hline Hernic & le compressive & $2 / 41(4,9)$ \\
\hline Myélopa & hon compressives & $30(42,3)$ \\
\hline Myéli & rculeuse & $9 / 30(30)$ \\
\hline Neuro & optique de Devic & $1 / 30(3,3)$ \\
\hline Encép & yélite aiguë disséminée & $1 / 30(3,3)$ \\
\hline Caren & vitamine $\mathrm{B} 12$ & $3 / 30(10)$ \\
\hline Tumel & dullaire & $1 / 30(3,3)$ \\
\hline Myéli & cause indéfinie & $15 / 30(50)$ \\
\hline
\end{tabular}


Sur le plan de la prise en charge thérapeutique, l'ensemble des 27 patients avec une tuberculose a bénéficié d'une quadrichimiothérapie (RHZE) spécifique comme dose d'attaque, puis d'un relais par une bithérapie (RH) selon le protocole de la tuberculose extrapulmonaire du Programme de lutte contre la tuberculose de la Côte-d'Ivoire. L'évolution clinique était favorable (sur les plans infectieux et fonctionnel, avec récupération motrice complète) chez sept patients avec une myélite tuberculeuse et huit patients avec une spondylodiscite à BK. Les trois cas de myélopathie avec carence en vitamine B12 ont bénéficié d'une supplémentation avec une très bonne évolution clinique.

Douze patients transférés en neurochirurgie et quatre en oncologie ont été perdus de vue.

Quelques complications de décubitus : cinq cas d'escarres, deux cas de thrombophlébite des membres et un cas de décès chez des patients avec des métastases.

\section{Discussion}

La fréquence hospitalière des myélopathies dans notre étude était de 7,1\%. À notre connaissance, notre travail représente la première étude hospitalière qui présente des données démographiques et étiologiques des myélopathies en Afrique subsaharienne. Toutes les études rapportées en Afrique subsaharienne ne décrivent qu'une étiologie bien déterminée de myélopathie $[1,4,9]$. Dans une étude systématique des cas de myélopathie non traumatique rapportés en Afrique subsaharienne, publiée récemment par Musubire et al. [5], les myélopathies compressives représentent $48 \%$ des cas et sont largement dominées par la spondylodiscite à BK et les métastases.

Dans notre étude, les patients âgés de 26 à 45 ans étaient les plus représentés $(40,8 \%)$, majoritairement de sexe masculin $(17 / 29=58,6 \%)$.

Ces résultats montrent que les pathologies médullaires affectent essentiellement l'adulte jeune de sexe masculin en pleine activité et sont à l'origine de conséquences socio-économiques et professionnelles parfois extrêmement importantes.

Les étiologies de myélopathie sont multiples dans la présente étude, avec une prédominance des causes infectieuses. Cela pourrait s'expliquer non seulement par la situation géographique de notre pays (zone tropicale), mais aussi par le niveau socioéconomique bas des populations.

La tuberculose était la principale cause des myélopathies. Cela pourrait s'expliquer par la persistance de l'infection à VIH, le brassage de populations lié à l'immigration et la précarité sociale [1]. L'association tuberculose et VIH a été retrouvée chez 11 patients. Les myélopathies liées au VIH sont fréquentes et sont dues soit à une atteinte directe de la moelle par le virus (myélopathie vacuolaire, myélite à VIH), soit à une infection opportuniste (myélite tuberculeuse, myélite virale ou parasitaire) [3]. La myélopathie a été le mode révélateur de l'infection à VIH chez $12,7 \%$ de nos patients.

Les myélopathies inflammatoires sont définies comme une atteinte inflammatoire de la moelle épinière de pathogénie hétérogène [8]. Elles représentent la catégorie nosologique la plus importante en Europe, aux États-Unis et au Maghreb [2]. Dans notre étude, nous avons retrouvé un cas d'EMAD et un cas de NMO séropositive. La NMO est rarement décrite en Afrique subsaharienne : la plus grande série a été rapportée par l'équipe d'Osuntokun au Nigeria (Ibadan) qui a colligé 95 cas de NMO en l'espace de 12 ans [7].

Dans notre travail, les myélites de cause indéterminée représentent la moitié des myélopathies non compressives. Quatre patients étaient VIH positif parmi ceux avec myélite de cause indéterminée. Chez ces patients, aucune infection opportuniste n'a été trouvée. Parmi les patients VIH négatif, quatre avaient un tableau clinique de myélopathie inflammatoire caractérisé par un mode évolutif en poussées-rémissions. La recherche des bandes oligoclonales ainsi que l'IRM cérébrale et le bilan immunologique systémique n'ont pas été réalisés chez l'ensemble des patients avec myélite de cause indéterminée. Chez les sept patients restants, le tableau clinique, paraclinique (étude du LCR) et évolutif (régression spontanée des signes cliniques) pouvait faire évoquer une myélite virale ou une première poussée de pathologie inflammatoire du SNC ou encore une myélite idiopathique. L'accès limité à certains examens biologiques pourrait expliquer le nombre important de myélopathies d'étiologie non déterminée.

\section{Conclusion}

Ce travail montre que dans le service de neurologie du CHU de Cocody les myélopathies sont fréquentes dans la pratique neurologique quotidienne.

- La moelle spinale thoracique était la plus atteinte ;

- la tuberculose était l'étiologie la plus fréquente dans les myélopathies ;

- aucun cas de sclérose en plaques n'a été diagnostiqué dans notre étude ;

- une prise en charge multidisciplinaire s'impose, afin d'optimiser l'offre de soins aux patients.

Liens d'intérêts : Les auteurs déclarent ne pas avoir de liens d'intérêts. 


\section{Références}

1. Bakayoko AS, Ouattara B, Kamagaté M, et al (2015) Traitement médical de la tuberculose vertébrale en Côte-d'Ivoire. Rev Pneumol Trop 23:70-6

2. Brochet B (2007) Myélopathies aiguës inflammatoires. Lett Neurol 6:123-6

3. Kennedy PG, Weir AI (1988) Rapid recovery of acute transverse myelitis treated with steroids. Postgrad Med J 64:384-5

4. Meftah M, Lmejjati M, Mansouri A, et al (2001) Mal de Pott : à propos de 320 cas. Med Maghreb 90:19-22

5. Musubire AK, Meya DB, Bohjanen PR, et al (2017) A systematic review of non-traumatic spinal cord injuries in Sub-Saharan
Africa and a proposed diagnostic algorithm for resource-limited settings. Front Neurol 8:618. doi: 10.3389/fneur.2017.00618. eCollection 2017

6. Myelopathy. Diseases Database Ver 1.8. Medical lists and links 2006 [en ligne] Consulté le 8 septembre 2017

7. Osuntokun BO (1971) The pattern of neurological illness in tropical Africa. Experience at Ibadan, Nigeria. J Neurol Sci 12:417-42

8. Pittock SJ, Lucchinetti CE (2006) Inflammatory transverse myelitis: evolving concepts. Curr Opin Neurol 19:362-8

9. Toloba Y, Diallo S, Maiga Y, et al (2011) Tuberculose vertébrale (mal de Pott) : aspects épidémioclinique, radiologique et évolutif au CHU du Point-G. Mali Med 26:8-11 